\title{
A facile four-component Gewald reaction under organocatalyzed aqueous conditions
}

\author{
M. Saeed Abaee* and Somayeh Cheraghi \\ Chemistry and Chemical Engineering Research Center of Iran, P.O.Box 14335-186, \\ Tehran, Iran \\ E-mail:abaee@ccerci.ac.ir
}

DOI: $\underline{\text { http://dx.doi.org/10.3998/ark.5550190.p008.427 }}$

\begin{abstract}
In the presence of water and triethylamine, a four-component process involving ethyl cyanoacetate, an $\alpha$-methylene carbonyl compound, a primary or a secondary amine, and elemental sulfur leads to efficient room-temperature formation of 2-amino-3-carboxamide derivatives of thiophene in short time periods. The products, which precipitate from the reaction mixtures, are easily obtained by simple filtration and recrystallization from ethyl acetate/hexanes.
\end{abstract}

Keywords: Gewald reaction, four-component reaction, aqueous conditions, 2-aminothiophenes, organocatalysis

\section{Introduction}

A multicomponent reaction (MCR) is defined as a process that causes combination of three or more reactants to form a product, exclusively or in adequate yield, in a one-pot operation. ${ }^{1,2}$ In an ideal case the process would take place highly selectively and a single product would form which retains all or the majority of the atoms of the reactants. These features have overwhelmingly led to numerous applications of MCRs in synthetic organic chemistry in recent years because MCRs allow direct access to complex target molecules and chemical libraries in a much more efficient and economical fashion. ${ }^{3,4}$

One of the best studied MCRs in recent decades has been the one-pot cyclocondensation of $\alpha$-methylene carbonyl compounds and $\beta$-substituted acetonitriles with elemental sulfur, ${ }^{5,6}$ a process which is named after its discoverer: the Gewald reaction. ${ }^{7}$ The 2-aminothiophene products of the reaction exhibit agrochemical, ${ }^{8}$ pharmaceutical, ${ }^{9}$ mesogenic, ${ }^{10}$ and dye ${ }^{11}$ properties. In several cases they are also the key substructure of organic structures ${ }^{12-14}$ and biologically active compounds. ${ }^{15,16}$ The original two-component Gewald reaction between $\alpha$ - 
mercaptoketones and cyanoacetate proceeds under basic catalysis. ${ }^{7}$ The many modifications reported so far extend the scope of this reaction by altering the components ${ }^{17-19}$ and the conditions. $^{20-23}$

Another interesting feature of the Gewald reaction is the use of the 2-aminothiophene products in other organic transformations. ${ }^{24,25}$ The growing use of MCRs in synthesis led us to examine the possibility of employing more than three components in the reaction so that we can access derivatized Gewald products in a one-pot operation. In the framework of our investigations on the development of one-pot synthetic procedures, ${ }^{26-28}$ we herein report a simple method for conducting a four-component Gewald reaction under inexpensive and benign aqueous triethylamine conditions. As a result of this method, reactions proceed within relatively short time periods and 2-aminothiophene- $N$-alkyl products precipitate spontaneously, perhaps because of high polarity of the medium, allowing their solvent-free separation from the reaction mixtures. The overall process is exemplified in Scheme 1 for the reaction of 3-phenylpropanal (1a) with methyl cyanoacetate (2), cyclohexanamine (3a), and elemental sulfur. It is noteworthy that a literature survey shows little precedent for a four-component strategy in the Gewald reaction. $^{29}$
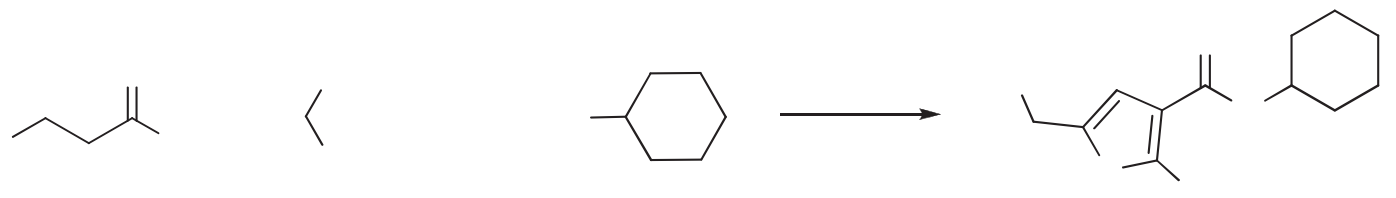

Scheme 1. Four-component synthesis of 4.

\section{Results and Discussion}

We first optimized the conditions by studying the reaction of $\mathbf{1 a}$ with $\mathbf{2}$, 3a, and sulfur at room temperature (Table 1). Among various secondary or tertiary amines used in the reaction (entries 1-5), $\mathrm{Et}_{3} \mathrm{~N}$ caused a higher conversion of the reactants into 4 . In addition, the use of $\mathrm{Et}_{3} \mathrm{~N}$ led to precipitation of the product at the end of the process (entry 1). Conversely, hexyl-1-amine gave negligible quantities of the product (entry 6). In the absence of the amine (entry 7) or water (entry 8) no formation of product was noticed, even after a longer time period, illustrating the promoting effect of both additives.

From a mechanistic point of view, when a reaction rate is enhanced under aqueous conditions, the acceleration is attributed to either the repulsive forces arising from hydrophobicity of the reactants ${ }^{30}$ or to activation of electron donating functional groups through H-bonding with water molecules. ${ }^{31}$ To study the role of these effects in the present work, we next altered the composition of the aqueous medium to investigate the effect of different additives on the progress of the reaction (entries 9-14). In these cases, an early work-up procedure was used (after $0.5 \mathrm{~h}$ ) so that a better comparison of the results could be concluded. Therefore, under 
optimum conditions $\left(\mathrm{H}_{2} \mathrm{O} / \mathrm{Et}_{3} \mathrm{~N}\right), 65 \%$ of 4 was formed after $0.5 \mathrm{~h}$ (entry 9). When solutions of $\mathrm{LiCl}$ (entry 10) or $\mathrm{NaCl}$ (entry 11) were used, yields increased, suggesting that hydrophobic forces are responsible for the rate enhancement in this Gewald reaction. This was further confirmed by observing a greater rate increase at a higher concentration of $\mathrm{NaCl}$ (entry 12). In contrast, a descending pattern was noticed for a similar reaction conducted in the presence of guanidinium chloride $(\mathrm{GnCl})$ (entry 13) or $\mathrm{LiClO}_{4}$ (entry 14). This excluded intervention of hydrogen-bonds as being a driving force for the reaction. Thus, one can conclude that hydrophobic behavior of the reactants in water provides the driving force of the reaction. On this basis, it can also be justified that why $\mathrm{Et}_{3} \mathrm{~N}$, which has the lowest solubility among the amines examined, causes a greater rate increase under the conditions, governed by hydrophobic effects.

Table 1. Optimization of the Gewald reaction for the synthesis of 4

\begin{tabular}{ccccc}
\hline Entry & Medium & Amine & Time $(\mathrm{h})$ & Yield $(\%)^{\mathrm{a}}$ \\
\hline 1 & $\mathrm{H}_{2} \mathrm{O}$ & $\mathrm{Et}_{3} \mathrm{~N}$ & 1 & 95 \\
2 & $\mathrm{H}_{2} \mathrm{O}$ & $\mathrm{DBU}$ & 1 & 88 \\
3 & $\mathrm{H}_{2} \mathrm{O}$ & $\mathrm{DABCO}$ & 1 & 81 \\
4 & $\mathrm{H}_{2} \mathrm{O}$ & morpholine & 1 & 72 \\
5 & $\mathrm{H}_{2} \mathrm{O}$ & $\mathrm{Et}_{2} \mathrm{NH}$ & 1 & 40 \\
6 & $\mathrm{H}_{2} \mathrm{O}$ & hexyl-1-amine & 1 & 7 \\
7 & - & $\mathrm{Et}_{3} \mathrm{~N}$ & 6 & 0 \\
8 & $\mathrm{H}_{2} \mathrm{O}$ & - & 6 & 0 \\
9 & $\mathrm{H}_{2} \mathrm{O}$ & $\mathrm{Et}_{3} \mathrm{~N}$ & 0.5 & 65 \\
10 & $\mathrm{LiCl}_{(\mathrm{aq}, 1.5 \mathrm{M})}$ & $\mathrm{Et}_{3} \mathrm{~N}$ & 0.5 & 68 \\
11 & $\mathrm{NaCl}(\mathrm{aq}, 1.5 \mathrm{M})$ & $\mathrm{Et}_{3} \mathrm{~N}$ & 0.5 & 70 \\
12 & $\mathrm{NaCl}(\mathrm{aq}, 3.0 \mathrm{M})$ & $\mathrm{Et}_{3} \mathrm{~N}$ & 0.5 & 75 \\
13 & $\mathrm{GnCl}(\mathrm{aq}, 1.5 \mathrm{M})$ & $\mathrm{Et}_{3} \mathrm{~N}$ & 0.5 & 30 \\
14 & $\mathrm{LiClO}_{4}(\mathrm{aq}, 1.5 \mathrm{M})$ & $\mathrm{Et}_{3} \mathrm{~N}$ & 0.5 & 33 \\
\hline
\end{tabular}

a isolated yields

Next, the generality of the method was investigated (Table 2). The optimum conditions under which 4 was formed in $95 \%$ yield and within $1 \mathrm{~h}$ (entry 1) were applied to the reactions of aldehydes 1a-b with various amines, 2, and sulfur. Thus, 92-97\% of products 5, 6, and $\mathbf{7}$ were formed after 1-2 $\mathrm{h}$ (entries 2-4). Then, the conditions were used for similar reactions of cyclohexanone 1c (entries 5-7). As a result, good to high yields of the respective products were obtained. For these entries, reaction times were relatively longer presumably due to lower reactivity of the starting ketone. Because of our interest in developing the chemistry of the 
thiopyran-4-one system, ${ }^{32,33}$ we subjected 1d to the reaction conditions and obtained the expected products as shown in entries 8-10.

Table 2. $\mathrm{H}_{2} \mathrm{O} / \mathrm{Et}_{3} \mathrm{~N}$ catalyzed four-component Gewald reactions

\begin{tabular}{|c|c|c|c|c|c|c|}
\hline Entry & $\begin{array}{l}\text { Ketone or } \\
\text { aldehyde }\end{array}$ & Amine & Product & $\begin{array}{l}\text { Time } \\
\text { (h) }\end{array}$ & $\begin{array}{c}\text { Yield }^{a} \\
(\%)\end{array}$ & $\operatorname{Mp}\left({ }^{\circ} \mathrm{C}\right)$ \\
\hline 1 & & & & 1 & 95 & $178-180$ \\
\hline 2 & & & & 2 & 92 & 164-166 \\
\hline 3 & & & & 1 & 97 & 191-193 \\
\hline 4 & & & & 2 & 95 & $195-197$ \\
\hline 5 & & & & 7 & 78 & $142-144$ \\
\hline 6 & & & & 7 & 81 & $113-115$ \\
\hline 7 & & & & 7 & 82 & $116-118$ \\
\hline 8 & & & & 5 & 77 & $131-133$ \\
\hline
\end{tabular}


Table 2. Continued

Entry $\begin{gathered}\text { Ketone or } \\ \text { aldehyde }\end{gathered}$ Amine

${ }^{a}$ isolated yields.

Based on these results, a mechanism, as depicted in Figure 1, can be suggested for the process. The starting amine, which is first mixed with $\mathbf{2}$, forms the corresponding amide. This amide then produces an $\alpha, \beta$-unsaturated nitrile intermediate via a Knoevenagel condensation. The Knoevenagel intermediate then reacts with sulfur to produce the final thiophene skeleton, after a ring closure and an aromatization rearrangement.

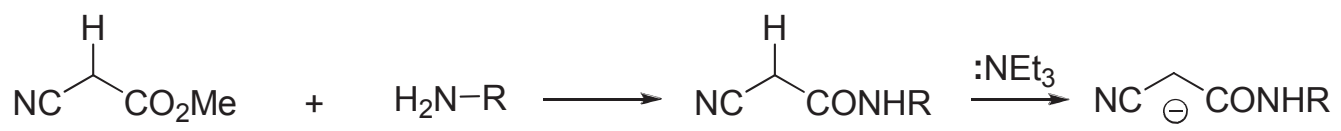<smiles>[X]CC(SSS)C(C#N)C(N)=O</smiles><smiles>NCCCS12C[SH]1SSSSSSS2</smiles>

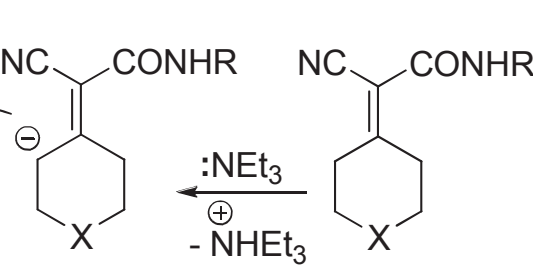<smiles>O=C1CCCCC1CCO</smiles>

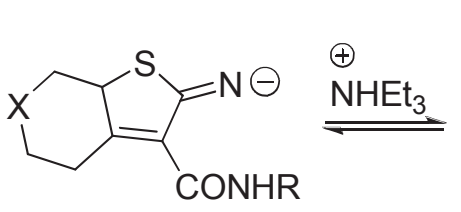

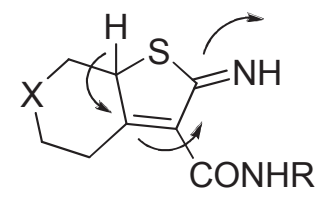<smiles>C1CCCCC1</smiles><smiles>[R]NC(=O)c1c(N)sc2c1CCC2</smiles>

Scheme 2. A suggested mechanism 


\section{Conclusions}

In summary, we have reported a general and efficient protocol for the preparation of various 2aminothiophene-3-carboxamide derivatives resulted from a four-component Gewald reaction. Thus, various ketones can combine with methyl cyanoacetate, an amine, and sulfur at room temperature in a one-pot process. Reactions occur using the $\mathrm{H}_{2} \mathrm{O} / \mathrm{Et}_{3} \mathrm{~N}$ environmentally safe medium, single products are rapidly formed in high yields, no complex operation or handling is required, and use of toxic organic solvents is avoided. These features make the present method an attractive addition to the present literature archive.

\section{Experimental Section}

General. Reactions were monitored by TLC using silica-gel coated plates and ethyl acetate/hexanes solutions as the mobile phase. Melting points are uncorrected. FT-IR spectra were recorded using $\mathrm{KBr}$ disks on a Bruker Vector-22 infrared spectrometer and absorptions are reported as wave numbers $\left(\mathrm{cm}^{-1}\right) .{ }^{1} \mathrm{H}$ NMR and ${ }^{13} \mathrm{C}$ NMR spectra were obtained on a FT-NMR Bruker Ultra Shield ${ }^{\mathrm{TM}}(500 \mathrm{MHz})$ instrument as $\mathrm{CDCl}_{3}$ solutions and the chemical shifts are expressed as $\delta$ units with $\mathrm{Me}_{4} \mathrm{Si}$ as the internal standard. Mass spectra were obtained on a Finnigan MAT 8430 apparatus at ionization potential of $70 \mathrm{eV}$. Elemental analyses were performed using a Thermo Finnigan Flash EA 1112 instrument. Compound 1d was prepared using available methods. ${ }^{34}$ All other chemicals were purchased from commercial sources and were used after being freshly purified by standard procedures. Products $4,7,10,{ }^{35}$ and $9^{16}$ are known. Other products were new and were characterized based on their spectral and physical data.

Typical procedure. A mixture of methyl cyanoacetate (2) (297 $\mathrm{mg}, 3 \mathrm{mmol}$ ) and cyclohexylamine (3a) ( $343 \mu \mathrm{L}, 3 \mathrm{mmol})$ was stirred at room temperature for 15 minutes. To this mixture was added 3-phenylpropionaldehyde (1a) $(400 \mu \mathrm{L}, 3 \mathrm{mmol})$, sulfur (96 mg, $3 \mathrm{mmol})$, water $(1.0 \mathrm{~mL})$, and $\mathrm{Et}_{3} \mathrm{~N}(418 \mu \mathrm{L}, 3.0 \mathrm{mmol})$ sequentially and stirring was continued at room temperature for another 45 minutes or until TLC showed complete disappearance of the starting materials. The product which solidified at the end of the reaction was separated by filtration and recrystallized from EtOAc/hexanes mixture. Product 4 was obtained in $95 \%$ yield $(895 \mathrm{mg})$. The product was identified based on its physical and spectral characteristics.

(2-Amino-5-benzylthien-3-yl)(4-phenylpiperazin-1-yl)methanone (5). Yellow solid, mp 164$166{ }^{\circ} \mathrm{C}$ (EtOAc/hexanes); ${ }^{1} \mathrm{H}$ NMR $\left(500 \mathrm{MHz}, \mathrm{CDCl}_{3}\right) \delta 3.23(\mathrm{t}, J 5.0 \mathrm{~Hz}, 4 \mathrm{H}), 3.83(\mathrm{t}, J 5.0 \mathrm{~Hz}$, 4H), 3.98 (s, 2H), 5.37 (br s, 2H), $6.45(\mathrm{~s}, 1 \mathrm{H}), 6.94-7.00(\mathrm{~m}, 3 \mathrm{H}), 7.26-7.30(\mathrm{~m}, 3 \mathrm{H}), 7.32-7.37$ $(\mathrm{m}, 4 \mathrm{H}) \mathrm{ppm} ;{ }^{13} \mathrm{C}$ NMR $\left(125 \mathrm{MHz}, \mathrm{CDCl}_{3}\right) \delta 36.4,45.5,50.2,109.8,117.0,121.0,123.1,126.4$, 127.1, 128.9, 129.0, 129.1, 129.7, 140.4, 159.4, 167.7 ppm; IR (KBr) 3387, 3290, 1624, 1597 
$\mathrm{cm}^{-1}$; MS $377\left(\mathrm{M}^{+}\right), 258,216,161,132$, 91; Anal. Calcd for $\mathrm{C}_{22} \mathrm{H}_{23} \mathrm{~N}_{3} \mathrm{OS}$ : C, 70.00; H, 6.14. Found: C, 70.24; H, 6.16\%.

2-Amino- $N$-cyclohexyl-5-phenylthiophene-3-carboxamide (6). Pink solid, mp 191-193 ${ }^{\circ} \mathrm{C}$ (EtOAc/hexanes); ${ }^{1} \mathrm{H}$ NMR (500 MHz, $\left.\mathrm{CDCl}_{3}\right) \delta 1.24-1.28$ (m, 3H) 1.43-1.46 (m, 2H), 1.67-1.69 $(\mathrm{m}, 1 \mathrm{H}), 1.77-1.81(\mathrm{~m}, 2 \mathrm{H}), 2.03-2.06(\mathrm{~m}, 2 \mathrm{H}), 3.90-3.93(\mathrm{~m}, 1 \mathrm{H}), 5.69(\mathrm{~s}, 1 \mathrm{H}), 5.72(\mathrm{br} \mathrm{s}, 2 \mathrm{H})$, 6.99 (s, 1H), $7.23(\mathrm{t}, J 7.5 \mathrm{~Hz}, 1 \mathrm{H}), 7.35(\mathrm{t}, J 7.5 \mathrm{~Hz}, 2 \mathrm{H}), 7.46(\mathrm{~d}, J 7.5 \mathrm{~Hz}, 2 \mathrm{H}) \mathrm{ppm} ;{ }^{13} \mathrm{C}$ NMR $\left(125 \mathrm{MHz}, \mathrm{CDCl}_{3}\right) \delta 25.5,26.0,33.9,48.5,110.9,118.5,125.1,126.2,127.1,129.3,134.4$, 159.8, 165.3 ppm; IR (KBr) 3439, 3298, 1608, $1571 \mathrm{~cm}^{-1}$; MS m/z $300\left(\mathrm{M}^{+}\right), 201,146,130$; Anal. Calcd for $\mathrm{C}_{17} \mathrm{H}_{20} \mathrm{~N}_{2} \mathrm{OS}$ : C, 67.97; H, 6.71. Found: C, 68.14; H, $6.66 \%$.

2-Amino- $N$-cyclohexyl-4,5,6,7-tetrahydrobenzo $[b]$ thiophene-3-carboxamide (8). Yellow solid, mp 142-144 ${ }^{\circ} \mathrm{C}$ (EtOAc/hexanes); ${ }^{1} \mathrm{H}$ NMR (500 MHz, $\left.\mathrm{CDCl}_{3}\right) \delta 1.18-1.24$ (m, 3H) 1.37$1.43(\mathrm{~m}, 2 \mathrm{H}), 1.59-1.62(\mathrm{~m}, 1 \mathrm{H}), 1.67-1.71$ (m, 2H), 1.78-1.80 (m, 4H), 1.94-2.03 (m, 2H), 2.52$2.54(\mathrm{~m}, 2 \mathrm{H}), 2.65-2.66(\mathrm{~m}, 2 \mathrm{H}), 3.88-3.95(\mathrm{~m}, 1 \mathrm{H}), 5.56(\mathrm{~d}, J 7.0 \mathrm{~Hz}, 1 \mathrm{H}), 6.00$ (br s, 2H) ppm; ${ }^{13} \mathrm{C} \mathrm{NMR}\left(125 \mathrm{MHz}, \mathrm{CDCl}_{3}\right) \delta 23.3,23.5,25.0,25.2,26.1,27.7,33.8,40.0,109.6,119.4,129.2$, 158.8, 166.0 ppm; IR (KBr) 3298, 1585, 1650, $1564 \mathrm{~cm}^{-1}$; MS m/z $278\left(\mathrm{M}^{+}\right), 196,179,151,83$; Anal. Calcd for $\mathrm{C}_{15} \mathrm{H}_{22} \mathrm{~N}_{2} \mathrm{OS}$ : C, 64.71; H, 7.96. Found: C, 64.87; H, 7.69\%.

2-Amino- $N$-cyclohexyl-5,7-dihydro-4H-thieno[2,3-c] thiopyran-3-carboxamide (11). Brown solid, mp 131-133 ${ }^{\circ} \mathrm{C}$ (EtOAc/hexanes); ${ }^{1} \mathrm{H}$ NMR (500 MHz, $\left.\mathrm{CDCl}_{3}\right) \delta$ 1.22-1.28 (m, 3H), 1.40$1.47(\mathrm{~m}, 2 \mathrm{H}), 1.62-1.66(\mathrm{~m}, 1 \mathrm{H}), 1.70-1.75(\mathrm{~m}, 2 \mathrm{H}), 1.97-2.00(\mathrm{~m}, 2 \mathrm{H}), 2.89-2.91(\mathrm{~m}, 4 \mathrm{H}), 3.65$ (s, 2H), 3.90-3.96 (m, 1H), $5.51(\mathrm{~d}, J 7.0 \mathrm{~Hz}, 1 \mathrm{H}), 6.00$ (br s, 2H) ppm; ${ }^{13} \mathrm{C}$ NMR $(125 \mathrm{MHz}$, $\left.\mathrm{CDCl}_{3}\right) \delta 25.2,25.7,26.0,26.4,29.3,33.7,48.3,111.2,115.6,129.7,157.3,165.5$ ppm; IR (KBr) 3361, 3298, 1635, 1590, $1564 \mathrm{~cm}^{-1}$; MS m/z $296\left(\mathrm{M}^{+}\right)$, 197, 170, 152, 41; Anal. Calcd for $\mathrm{C}_{14} \mathrm{H}_{20} \mathrm{~N}_{2} \mathrm{OS}_{2}$ : C, 56.72; H, 6.80. Found: C, 56.61; H, $6.73 \%$.

(2-Amino-5,7-dihydro-4H-thieno[2,3-c]thiopyran-3-yl)(4-phenylpiperazin-1-yl)methanone (12). White solid, mp 155-157 ${ }^{\circ} \mathrm{C}$ (EtOAc/hexanes); ${ }^{1} \mathrm{H}$ NMR $\left(500 \mathrm{MHz}, \mathrm{CDCl}_{3}\right) \delta 2.68-2.78$ (m, $2 \mathrm{H}), 2.79-2.87(\mathrm{~m}, 2 \mathrm{H}), 2.88-2.92(\mathrm{~m}, 2 \mathrm{H}), 2.93-2.95(\mathrm{~m}, 2 \mathrm{H}), 3.21-3.23(\mathrm{~m}, 4 \mathrm{H}), 3.67(\mathrm{~s}, 2 \mathrm{H})$, $3.84(\mathrm{~s}, 2 \mathrm{H}), 6.93-6.96(\mathrm{~m}, 3 \mathrm{H}), 7.25-7.31(\mathrm{~m}, 2 \mathrm{H}) \mathrm{ppm} ;{ }^{13} \mathrm{C} \mathrm{NMR}\left(125 \mathrm{MHz}, \mathrm{CDCl}_{3}\right) \delta 31.0$, 34.6, 36.5, 42.5, 47.3, 50.0, 50.3, 106.5, 114.8, 117.4, 121.5, 128.0, 129.8, 151.0, 161.2, 164.7 ppm; IR (KBr) 3265, 3062, 2823, 1637, 1498, $1026 \mathrm{~cm}^{-1}$; MS m/z $359\left(\mathrm{M}^{+}\right), 327,278,179,132$; Anal. Calcd for $\mathrm{C}_{18} \mathrm{H}_{21} \mathrm{~N}_{3} \mathrm{OS}_{2}$ : C, 60.14; H, 5.89. Found: C, 60.11; H, $5.75 \%$.

2-Amino- $N$-benzyl-5,7-dihydro-4H-thieno[2,3-c]thiopyran-3-carboxamide (13). Brown solid, mp 130-134 ${ }^{\circ} \mathrm{C}$ (EtOAc/hexanes); ${ }^{1} \mathrm{H}$ NMR (500 MHz, $\left.\mathrm{CDCl}_{3}\right) \delta 2.85-2.87$ (m, 2H), 2.882.92 (m, 2H), 3.63 (s, 2H), 4.58 (d, J 7.0 Hz, 2H), 5.88 (br s, 2H), 5.97 (t, J 7.0 Hz, 1H), 7.297.39 (m, 5H) ppm; ${ }^{13} \mathrm{C}$ NMR $\left(125 \mathrm{MHz}, \mathrm{CDCl}_{3}\right) \delta 25.7,26.3,29.3,43.9,110.4,115.5,127.9$, 128.1, 129.2, 129.7, 139.0, 158.1, 166.4 ppm; IR (KBr) 3265, 3062, 2823, 1637, 1498, $1026 \mathrm{~cm}^{-}$

1; MS m/z, $304\left(\mathrm{M}^{+}\right)$, 197, 170, 152, 104, 91; Anal. Calcd for $\mathrm{C}_{15} \mathrm{H}_{16} \mathrm{~N}_{2} \mathrm{OS}_{2}$ : C, 59.18; H, 5.30. Found: C, 59.33; H, $5.41 \%$. 


\section{Acknowledgements}

Partial financial support by the Ministry of Science, Research, and Technology of Iran is gratefully appreciated.

\section{References}

1 Dömling, A.; Wang, W.; Wang, K. Chem. Rev. 2012, 112, 3083.

http://dx.doi.org/10.1021/cr100233r PMid:22435608 PMCid:PMC3712876

2 Perreault, S.; Rovis, T. Chem. Soc. Rev. 2009, 38, 3149.

http://dx.doi.org/10.1039/b816702h

PMid:19847348 PMCid:PMC2893402

3 Zhu, J.; Bienaymé, H. Multicomponent Reactions, Wiley-VCH: Weinheim, 2005. http://dx.doi.org/10.1002/3527605118

4 Akritopoulou-Zanze, I.; Djuric, S. W. in Synthesis of Heterocycles via Multicomponent Reactions II; Orru, R. V. A.; Ruijter, E. Eds.; Springer: New York, 2010; pp. 231-287. http://dx.doi.org/10.1007/7081_2010_46

5 Huang, Y.; Dömling, A. Mol. Divers. 2011, 15, 3. http://dx.doi.org/10.1007/s11030-010-9229-6 PMid:20191319

6 Puterová, Z.; Krutošíková, A.; Végh, D. Arkivoc 2010, (i), 209. http://dx.doi.org/10.3998/ark.5550190.0011.105

7 Gewald, K. Angew. Chem. 1961, 73, 114. http://dx.doi.org/10.1002/ange.19610730307

8 Sabnis, R. W.; Rangnekar, D. W.; Sonawane, N. D. J. Heterocycl. Chem. 1999, 36, 333. http://dx.doi.org/10.1002/jhet.5570360203

9 Wang, W.; Shangguan, S.; Qiu, N.; Hu, C.; Zhang, L.; Hu, Y. Bioorg. Med. Chem. 2013, 21, 2879. http://dx.doi.org/10.1016/j.bmc.2013.03.061

PMid:23601819

10 Puterová, Z.; Romiszewski, J.; Mieczkowski, J.; Gorecka, E. Tetrahedron 2012, 68, 8172. http://dx.doi.org/10.1016/j.tet.2012.07.075

11 Hallas, G.; Towns, A. D. Dyes Pigments 1997, 35, 219. http://dx.doi.org/10.1016/S0143-7208(96)00111-8

12 Behbehani, H.; Ibrahim, H. M.; Makhseed, S.; Elnagdi, M. H.; Mahmoud, H. Eur. J. Med. Chem. 2012, 52, 51.

http://dx.doi.org/10.1016/j.ejmech.2012.03.004

PMid:22464424 
13 Yamuna, E.; Zeller, M.; Adero, P. O.; Prasad, K. J. R. Arkivoc 2012, (vi), 326. http://dx.doi.org/10.3998/ark.5550190.0013.630

14 Fondjo, E. S.; Döpp, D.; Henkel, G. Tetrahedron 2006, 62, 7121. http://dx.doi.org/10.1016/j.tet.2006.04.037

15 Aurelio, L.; Figler, H.; Flynn, B. L.; Linden, J.; Scammells, P. J. Bioorg. Med. Chem. 2008, $16,1319$.

http://dx.doi.org/10.1016/j.bmc.2007.10.065

PMid:17980606

16 Nikolakopoulos, G.; Figler, H.; Lindenb, J.; Scammells, P. J. Bioorg. Med. Chem. 2006, 14, 2358.

http://dx.doi.org/10.1016/j.bmc.2005.11.018

PMid:16314104

17 Huang, Y.; Dömling, A. Chem. Biol. Drug Des. 2010, 76, 130.

http://dx.doi.org/10.1111/j.1747-0285.2010.00990.x

PMid:20545946 PMCid:PMC2913473

18 Thomae, D.; Perspicace, E.; Henryon, D.; Xu, Z.; Schneider, S.; Hesse, S.; Kirsch, G.; Seck, P. Tetrahedron 2009, 65, 10453. http://dx.doi.org/10.1016/j.tet.2009.10.021

19 Puterová, Z.; Andicsová, A.; Végh, D. Tetrahedron 2008, 64, 11262. http://dx.doi.org/10.1016/j.tet.2008.09.032

20 Tayebee, R.; Javadi, F.; Argi, G. J. Mol. Catal. A Chem. 2013, 368-369, 16. http://dx.doi.org/10.1016/j.molcata.2012.11.011

21 Zhao, D.-D.; Li, L.; Xu, F.; Wu, Q.; Lin, X.-F. J. Mol. Catal. B Enzym. 2013, 95, 29. http://dx.doi.org/10.1016/j.molcatb.2013.05.014

22 Castanedo, G. M.; Sutherlin, D. P. Tetrahedron Lett. 2001, 42, 7181. http://dx.doi.org/10.1016/S0040-4039(01)01470-8

23 Revelant, G.; Dunand, S.; Hesse, S.; Kirsch, G. Synthesis 2011, 43, 2935. http://dx.doi.org/10.1055/s-0030-1261032

24 Barnes, D. M.; Haight, A. R.; Hameury, T.; McLaughlin, M. A.; Mei, J.; Tedrow, J. S.; Riva Toma, J. D. Tetrahedron 2006, 62, 11311. http://dx.doi.org/10.1016/j.tet.2006.07.008

25 Rádl, S.; Obadalová, I. Arkivoc 2005, (xv), 4. http://dx.doi.org/10.3998/ark.5550190.0006.f02

26 Abaee, M. S.; Cheraghi, S.; Navidipoor, S.; Mojtahedi, M. M.; Forghani, S. Tetrahedron Lett. 2012, 53, 4405. http://dx.doi.org/10.1016/j.tetlet.2012.06.040

27 Abaee, M. S.; Mojtahedi, M. M.; Saberi, F.; Karimi, G.; Rezaei, M. T.; Mesbah, A. W.; Harms, K.; Massa, W. Synlett 2012, 23, 2073. http://dx.doi.org/10.1055/s-0031-1290438 
28 Mojtahedi, M. M. Abaee, M. S.; Mahmoodi, P.; Adib. M. Synth. Commun. 2010, 40, 2067. http://dx.doi.org/10.1080/00397910903219435

29 Yao, R.; Xia, E.; Sun, J.; Yan, C. Chinese J. Chem. 2011, 29, 2461. http://dx.doi.org/10.1002/cjoc.201180417

30 Breslow, R. Acc. Chem. Res. 2004, 37, 471.

http://dx.doi.org/10.1021/ar040001m

PMid:15260509

31 Lindström, U. M. Chem. Rev. 2002, 102, 2751.

http://dx.doi.org/10.1021/cr010122p

PMid:12175267

32 Abaee, M. S.; Mojtahedi, M. M.; Akbari, M.; Mehraki, E.; Mesbah, A. W.; Harms, K. J. Heterocycl. Chem. 2012, 49, 1346.

http://dx.doi.org/10.1002/jhet.976

33 Mojtahedi, M. M.; Abaee, M. S.; Khakbaz, M.; Alishiri, T.; Samianifard, M.; Mesbah, A. W.; Harms, K. Synthesis 2011, 43, 3821.

http://dx.doi.org/10.1055/s-0031-1289571

34 Ward, D. E.; Rasheed, M. A.; Gillis, H. M.; Beye, G. E.; Jheengut, V.; Achonduh, G. T. Synthesis 2007, 39, 1584.

http://dx.doi.org/10.1055/s-2007-965954

35 Wang, K.; Kim, D.; Dömling, A. J. Comb. Chem. 2010, 12, 111.

http://dx.doi.org/10.1021/cc9001586

PMid:19958011 PMCid:PMC3721980 